\title{
Strategy switches in proactive inhibitory control and their association with task-general and stopping-specific networks
}

\author{
Mari S. Messel ${ }^{\mathrm{a}, \mathrm{b}, \mathrm{c}}$, Liisa Raud ${ }^{\mathrm{a}, \mathrm{b}}$, Per Kristian Hoff ${ }^{\mathrm{a}}$, Cecilie Sol Skaftnes ${ }^{\mathrm{a}, \mathrm{b}}$, \\ René J. Huster ${ }^{\mathrm{a}, \mathrm{b}}$
}

\begin{abstract}
${ }^{\text {a }}$ Multimodal Imaging and Cognitive Control Lab, Department of Psychology, University of Oslo, Oslo, Norway.

${ }^{\mathrm{b}}$ Cognitive Electrophysiology Cluster, Department of Psychology, University of Oslo, Oslo, Norway.

${ }^{c}$ Sunnaas Rehabilitation Hospital, Nesodden, Norway
\end{abstract}

Corresponding author: René J. Huster, rene.huster@psykologi.uio.no 
Abstract

Prior information about the likelihood of a stop-signal pre-activates networks associated with response inhibition in both go- and stop-trials. How such prior information modulates the neural mechanisms enacting response inhibition and those balancing task strategies is only poorly understood. To investigate this, a cued stop-signal task (with cues indicating stopping probabilities of $0 \%, 25 \%$ or $66 \%$ ) was implemented in combination with functional magnetic resonance imaging (fMRI) data acquisition. Specifically, we focused on the effect of proactive inhibitory control as reflected in the activity of regions known to regulate response inhibition. Further, modulatory activity profiles in three different sub-regions of the right inferior frontal area were investigated. Behavioural results revealed an adaptation of task strategies through proactive control, with a possible gain for efficient inhibition at high stopping probabilities. The imaging data indicate that this adaption of task strategies was supported by different regions traditionally involved in the stopping network. While the right inferior parietal cortex (IPC), right middle frontal gyrus (MFG), right inferior frontal gyrus (rIFG) pars triangularis, and left anterior insula all showed increased go-trial activity in the $0 \%$ condition compared to the $25 \%$ condition, the pre-supplementary motor area (pre-SMA), anterior midcingulate cortex (aMCC), right anterior insula, and the rIFG pars opercularis showed a more stopping-specific pattern, with stronger stop-trial activity in the $66 \%$ condition than in the $25 \%$ condition. Furthermore, activity in inferior frontal sub-regions correlated with behavioural changes, where more pronounced response slowing was associated with stronger activity increases from low to high stopping probabilities. Notably, the different right inferior frontal sub-regions showed different activity patterns in response to proactive inhibitory control modulations, supporting the idea of a functional dissociation within this area. Specifically, while the pars opercularis and the right insula showed stopping-related modulations of activity, the rIFG pars triangularis exhibited modulations only in go-trials with strong adaptions to fast responding or proactive slowing. Overall, the results indicate that proactive inhibitory control results in the switching of task or strategy modes, either favouring fast responding or stopping, and that these strategical adaptations are governed by an interplay of different regions of the stopping network.

Keywords: fMRI, response inhibition, proactive control 


\section{Introduction}

Response inhibition refers to the ability to suppress or cancel a routine, prepotent or already initiated action (Hampshire \& Sharp, 2015). This ability is critical for the adaption of thoughts and behaviour to a rapidly changing environment, and is one of the hallmarks of cognitive control (Miyake et al., 2000). While response inhibition traditionally has been investigated as the process triggered by the sudden onset of a stop-signal, so-called reactive inhibition, research has indicated that how much one prepares to stop, so-called proactive control of inhibition, may affect the neural mechanisms enacting response inhibition (Aron, 2011; Verbruggen \& Logan, 2009b). While reactive inhibitory control is a bottom-up process related to the detection of a stop-signal, the modulation of inhibition through proactive control, henceforth referred to as proactive inhibitory control, is a top-down, workingmemory dependent process associated with goal-directed behaviour (Braver, 2012). These two modes of inhibitory control supposedly interact through neural systems to facilitate and balance fast and effective inhibition according to environmental demands (Aron, 2011). Here, we investigated the parametric effect of proactive inhibitory control on conditions requiring reactive inhibitory control (i.e., stop-trials) and conditions without an explicit need for stopping (i.e., go-trials). In addition, we identified brain regions associated with proactive inhibitory control by correlation analysis with behavioural indices, and further investigated connectivity changes associated with modulations of proactive inhibitory control. Not least, based on recent debates regarding the neural correlates of response inhibition in the right inferior frontal area, we considered a putative functional dissociation of this area. Specifically, we investigated the modulatory effects of proactive inhibitory control on three different regions of the inferior frontal area, namely the right inferior frontal gyrus (rIFG) pars opercularis, rIFG pars triangularis, and the right anterior insula.

The stop-signal task (SST) is probably the most widely used paradigm to study response inhibition, not least because of the possibility to derive an estimate of the stopping latency from it. This stopping latency is approximated by the stop-signal reaction time (SSRT), computed as a difference measure of a go-trial reaction time distribution parameter (e.g., mean or median) and the stop-signal delay (SSD; Band et al., 2003; Verbruggen \& Logan, 2009a). The use of the SST in combination with fMRI has consistently revealed activity associated with reactive inhibitory control in the bilateral IFG, the bilateral insula, the bilateral middle frontal gyrus (MFG), the dorsal anterior cingulate cortex (dACC) or 
midcingulate cortex (MCC) and the pre-supplementary motor area (pre-SMA), in addition to more posterior activations in the parietal cortex (reviewed in Aron et al., 2014; van Belle et al., 2014; Levy \& Wagner, 2011). However, the different functional contributions of these regions in context of reactive inhibitory control have remained equivocal. Some research has pointed to the rIFG as an important region for inhibitory control in the brain (reviewed in Aron, 2007; Aron et al., 2014), supposedly modulating the basal ganglia directly or via the pre-SMA in a right-lateralised network. Alternatively, it has been suggested that the rIFG and pre-SMA are part of a wider cortical network, in which each region has flexible functional roles contributing to a wide variety of cognitive tasks beyond mere inhibitory control (reviewed in Hampshire \& Sharp, 2015; Mirabella, 2014).

In the stop-signal paradigm, inhibitory control can be modulated proactively by explicitly informing the participant about the likelihood of an upcoming stop-signal. Thus, while reactive response inhibition is triggered by a somewhat surprising stop-signal, proactive inhibitory control is elicited by the probability cue indicating the likely occurrence of a stopsignal. Increased stop-signal probability has been associated with increased go-trial reaction times (go-RTs; Albares et al., 2014; Verbruggen \& Logan, 2009b; Zandbelt \& Vink, 2010). This behavioural adjustment is in line with adaptions of the speed-accuracy trade-off as a consequence of higher proactive control, where speed in go-trials is traded for successful inhibition in stop-trials (Forstmann et al., 2010; Verbruggen \& Logan, 2008). Further, contrasting fMRI activations of go-trials where the probability of a stop-signal is high (uncertain go-trials), and go-trials where the probability of a stop-signal is zero (certain gotrials) has revealed increased activity in several regions associated with reactive inhibitory control, such as the dorsolateral prefrontal cortex (DLPFC; Chikazoe et al., 2009; Swann et al., 2013), insula (Jahfari et al., 2012), pre-SMA (Albares et al., 2014; Chikazoe et al., 2009; Jahfari et al., 2012; Swann et al., 2012) and the rIFG (Jahfari et al., 2012; Swann et al., 2012). Since go-trials do not include a stop-signal, and thus should not elicit reactive inhibitory control, this activity has been interpreted as reflecting an early, proactive activation of the otherwise reactive inhibitory network (Jaffard et al., 2008).

In addition to the comparison of uncertain and certain go-trials, recent research has also looked at the parametric effects of increasing stop-signal probability on the activity in the stopping network. Such a manipulation yields the possibility to investigate whether increasing stop-signal probability leads to a corresponding increase in activity across the stopping network. Interestingly, manipulating the degree of go-uncertainty has revealed that not all 
regions implicated in response inhibition show the same modulation of activity (Leunissen et al., 2016; Zandbelt et al., 2013). It may be that the activity modulations seen in response to such parametric manipulations depend on whether the activity is associated with outright stopping tied to the stop-signal, or to the proactive inhibitory control processes as elicited by the probability cue (Zandbelt et al., 2013), although no consensus has been reached. This may partly be because there has only recently been a shift towards the investigation of modulatory effects of varying stop-signal probability beyond the mere binary comparison of certain and uncertain go-trials. One has to note that activity changes in any brain region may thus reflect the engagement of task-general processes, task-specific (here stopping-specific) processes, or a mixture of the two, and broader parametric manipulations may be necessary to disentangle these effects.

The effect of proactive inhibitory control on response inhibition can putatively manifest itself in a number of ways. First, proactive inhibitory control may modulate the inhibition network already before the presentation of a stop signal, for example by heightening response thresholds (Jahfari et al., 2010). Secondly, it may facilitate the detection of the stop signal, e.g. through the adaptation of attentional processes associated with the novelty or unexpectedness of the stop-signal (Gur et al., 2007; Shulman et al., 2009; Zandbelt et al., 2013). Thirdly, proactive inhibitory control processes may be manifested by regulating the reactive inhibition network after the stop signal has been detected, possibly via direct effects on the fronto-basal ganglia connections (Aron, 2011; Aron \& Poldrack, 2006; Jahfari et al., 2012). For example, it has been suggested that while reactive inhibitory control might be implemented via a hyperdirect pathway through the subthalamic nucleus (STN) of the basal ganglia, conditions of proactive inhibitory control may rather invoke pathways via a more indirect, slower route through the caudate (Aron, 2011). Activity in basal ganglia regions may thus also be differentially affected in accordance with the degree of proactive inhibitory control (Leunissen et al., 2016).

Another recent debate relates to the functional unity of the rIFG and adjacent regions (e.g., Aron, 2011; Erika-Florence et al., 2014; Hampshire et al., 2010; Swick \& Chatham, 2014; Verbruggen et al., 2010). It has been argued, for example, that analyses using a rIFG region of interest (ROI) located in medial parts of the rIFG might overlap with the anterior insula (Hampshire et al., 2010), and results may thus be contaminated by insular activity. This is problematic as it has been noted that insular activity during stop-trials might reflect autonomic arousal related to stopping (Aron et al., 2014), or the detection of behaviourally 
salient events (Cai et al., 2014). Further, whether the rIFG activity during response inhibition tasks reflects attentional processes tied to the detection of the stop-signal (Hampshire et al., 2010), inhibition related processes beyond mere attentional capture (Sebastian et al., 2016), or whether the rIFG sub-regions are part of different cortical networks supporting inhibition as well as other, more general cognitive control functions (Erika-Florence et al., 2014), is still unresolved. Recently, Hartwigsen et al. (2018) proposed a further functional segregation of the rIFG across the anterior-posterior axis, with the posterior parts being associated with action and inhibition-related functions, whereas the anterior parts were rather associated with cognition-related functions. Whether the neural correlates of response inhibition are indeed stronger associated with posterior and lateral structural parts of the rIFG (Aron et al., 2007, Swann et al., 2012; Wessel et al., 2013) needs further investigation.

Although previous research suggests that at least part of the stopping network is recruited during conditions of proactive inhibition, the effects of the modulation of proactive inhibitory control on the activity of this network still remains equivocal on at least two aspects. First, how is the stopping network associated with reactive inhibitory control and its parametric modulation by stop-signal probabilities (as opposed to an only binary comparison of uncertain go-trials and certain go-trials)? Second, does this modulation differentially affect sub-regions of the right inferior frontal area? To this means, a cued stop-signal task was implemented in combination with fMRI data acquisition. The cued stop-signal task included three stop-signal probabilities: $0 \%, 25 \%$ and $66 \%$. To investigate the modulatory effects of proactive inhibitory control on regions supposedly involved in inhibition, nine ROIs were defined: left and right insula, pre-SMA, anterior midcingulate cortex (aMCC), right IPC, right MFG, right IFG pars opercularis (hereby referred to as pars opercularis), right IFG pars triangularis (hereby referred to as pars triangularis), and the left caudate of the striatum. These regions were chosen in accordance with the meta-analysis of Cieslik et al. (2015). While the pars opercularis is the region traditionally implicated in response inhibition, including the pars triangularis makes it possible to investigate whether rIFG sub-regions are modulated differently by varying degrees of proactive control. It has to be noted that the right anterior insula region defined here overlaps slightly with the rIFG regions as reported in other studies, such as the one of Hampshire et al. (2010). Thus, these three regions (right anterior insula, pars opercularis, and pars triangularis) represent previous findings in the response inhibition literature and different sides of the debate regarding the functional specificity of the right inferior frontal area. 
Although cued stop-signal tasks make it possible to elicit proactive inhibitory control processes, it becomes more difficult to temporally dissociate cue-related activity from activity elicited by the subsequent go- and stop-signals. Thus, the hypotheses regarding go- and stoptrial activity will rely on the expected observed net effect of cue- and signal-associated activity. In go-trials, we expected an increase in cue- and go-signal activity with increasing stop-signal probability in regions associated with the top-down allocation of attention, working memory and task rules as well as the regions involved in stopping, thus reflecting the engagement of proactive inhibitory control as a top-down goal-directed process (Braver, 2012). This increase should therefore be evident in the IFG, MFG and IPC, as well as in the pre-SMA, as a result of the proactive preparation of stopping. Behaviourally, this activity increase was expected to be paralleled by an increase in go-trial reaction times.

In stop-trials, the observed net activity would reflect the proactive inhibitory control processes elicited by the cue, as well as reactive inhibitory control processes elicited by the stop-signal. It was thus hypothesized that in the $25 \%$ condition, the cue-related activity would reflect a middle-ground between low and high proactive inhibitory processes, while the reactive inhibitory processes would be relatively high. In the $66 \%$ stop condition, on the other hand, proactive inhibitory control as elicited by the cue would be high, while the reactive inhibitory control as elicited by the stop-signal would be relatively low, as a result of altered response or inhibition thresholds via the basal ganglia (Jahfari et al., 2010; Jahfari et al., 2012). Thus, regions more closely related to outright stopping, such as the IFG and the preSMA would in sum show mid-level elevated stop-related activity in both the $25 \%$ and $66 \%$ condition due to the differential and partly countermanding effects of proactive and reactive inhibitory control. Regions, on the other hand, that are not directly involved in reactive stopping but that have rather been associated with general attentional control (top-down or proactive), such as the MFG and IPC, would be expected to exhibit an increase in net activity with increasing stop-signal probability. Regarding the three sub-regions in the right inferior frontal area (i.e., pars triangularis, pars opercularis, and right anterior insula), any differential effect of the modulation of stop-signal probability on these regions would indicate functional specificity within the right inferior frontal area.

\section{Materials and Methods}

\subsection{Participants}


A total of 39 participants were recruited for the study. All participants reported normal or corrected-to-normal vision and no neurological or psychiatric disorders. Eleven participants were excluded from further data analysis; five due to excessive movement in the scanner, one aborted the experiment due to discomfort during scanning, and one participant reported lefthandedness that was not noticed until after the completion of the experiment. Four participants were excluded based on behavioural results showing a stop-trial accuracy below $20 \%$ (one participant) or above $80 \%$ (three participants). This left a final sample of twentyeight healthy, right-handed participants (age: range $=20-39$ years, $M=24.68, S D=4.03 ; 20$ female). The experiment was approved by the local ethics committee at the Department of Psychology, University of Oslo. Prior to participating in the study, all participants read and signed an informed consent form, and were informed that they could withdraw from the study at any time. Participants received a compensation of 200 NOK each for their participation.

\subsection{Task}

A cued stop-signal task with three levels of stop-signal probabilities was implemented in combination with fMRI data acquisition. The cued stop-signal task had a jittered, eventrelated design optimised for fMRI scanning. A visual representation of the task is presented in Figure 1. The task consisted of 600 task-trials in total. These were divided over three different cue conditions: $0 \%, 25 \%$ and $66 \%$. Specifically, the $0 \%$ condition consisted of 50 go-trials, the $25 \%$ condition of 300 go-trials and 100 stop-trials, and the $66 \%$ condition of 50 go-trials and 100 stop-trials. The overall percentage of go- relative to stop-trials thus was $2 / 1(\sim 33 \%$ stop trials); this ratio was chosen to still guarantee a prepotent tendency towards fast responding, and correspondingly a high load on motor inhibition. In addition to the 600 tasktrials (i.e., go- and stop-trials), 150 null-event trials were added to the design. These events consisted of a 1500 millisecond presentation of the fixation cross.

Each task-trial started with a fixation cross with a duration jittered randomly between 500-2400 milliseconds. The fixation cross was followed by a cue indicating the stop-signal probability of a given trial (i.e., $0 \%, 25 \%$ or $66 \%$ ), with a cue duration jittered randomly between 1000-2000 milliseconds. After the presentation of the cue, the go-signal was presented for 100 milliseconds. In go-trials, the go stimulus offset was followed by a fixation cross for another 1400 milliseconds, during which the responses were collected. Responses outside this timeframe were logged as no-responses. Go-signals consisted of either a left- or 
right-ward pointing green arrow, indicating the response-hand to be used (left or right, respectively).

The stop-trials were identical to the go-trials with one exception; in stop-trials, a blue arrow (i.e., the stop-signal) pointing in the same direction as the preceding go-signal was presented for 100 milliseconds with varying delays with respect to the go signal (SSD). For each participant, the SSD started at 300 milliseconds and was then dynamically adjusted using a staircase procedure to ensure a 50\% stop-trial accuracy. That is, the SSD was increased by 50 milliseconds (adjusted to the screen refresh rate) if the previous stop-trial resulted in successful inhibition (i.e., no response), and decreased by 50 milliseconds if the previous stop-trial resulted in unsuccessful inhibition (i.e., a response). The SSD was adjusted separately for each cue-condition, but collapsed for the right and left hand.

The 750 trials (task trials and null-event trials) were divided into 10 experimental blocks, with a randomised presentation of trials regarding left- and right-ward pointing arrows, cue-condition, go- and stop- conditions, and null-events. Each block lasted for approximately six minutes. After each block, participants were given feedback on their behavioural performance. If the average go-RT for that block was above 600 milliseconds, participants were presented the feedback "be faster", and if the average go-RT was below 600 milliseconds the participants were presented the feedback "well done". The overall duration of the task was approximately one hour.

a)

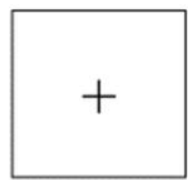

ITI

*500-2400 ms

b)

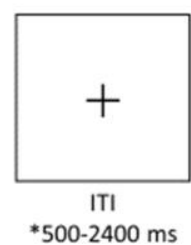

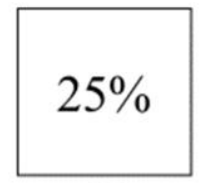

cue
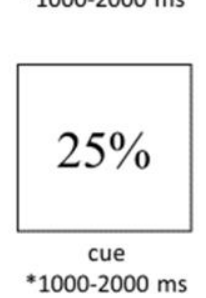
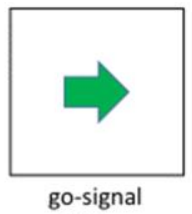
$100 \mathrm{~ms}$

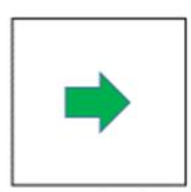

go-signal
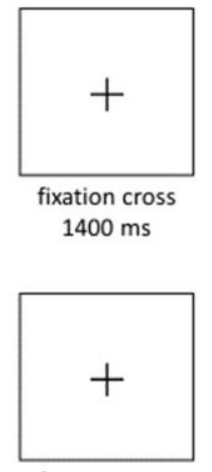

fixation cross SSD

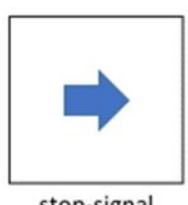

stop-signal

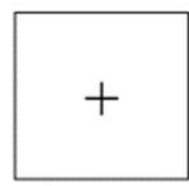

fixation cross $1400 \mathrm{~ms}-$ SSD

Figure 1: Visual representation of the cued stop-signal task. This figure illustrates an example of a go-trial (a) and stop-trial (b) with $25 \%$ stop-signal probability and rightwards pointing 
arrows. ITI $=$ inter-trial interval, $m s=$ milliseconds, $*$ indicates that the duration was jittered randomly within the specified time interval.

\subsection{Image Acquisition}

Structural and functional MR data were acquired from a 3.0 Tesla Philips Ingenia whole body MR scanner, equipped with a 32-channel Philips SENSE head coil (Philips Medical Systems, Best, the Netherlands). Each session started with a structural high resolution image using a T1-weighted sequence of 184 sagittal slices with a voxel size of 1 x 1 x $1 \mathrm{~mm}$, (field of view: 256 × $256 \mathrm{~mm}^{2}$, acquisition matrix: 256 x 256, TE: $2.2 \mathrm{~ms}$, TR: $4.5 \mathrm{~ms}$, flip angle: $8^{\circ}$, no slice gap). The fMRI sequence was a BOLD-sensitive T2* weighted echo-planar imaging (EPI) sequence of 34 axial slices with a voxel size of $2.625 \times 2.625 \times 3.0 \mathrm{~mm}$ (field of view: $210 \times$ $210 \mathrm{~mm}^{2}$, acquisition matrix: $80 \times 80$, TE: $30 \mathrm{~ms}$, TR: $2000 \mathrm{~ms}$, flip angle: $80^{\circ}$, slice gap: 0.3 $\mathrm{mm}$, interleaved acquisition). At the beginning of the fMRI sequence, three dummy scans were acquired and then discarded to allow for the stabilization of the magnetic field. The whole MRI session, including calibration, structural and functional sequences, took approximately one hour and ten minutes. Concurrent EEG data was recorded, but only the fMRI data is analyzed and discussed here.

\subsection{Analysis}

2.4.1 Behavioural data. For each participant, go-RT, go accuracy, SSRT, unsuccessful stoptrial reaction time (US-RT; i.e., the response time of stop-trial errors of commission), SSD, and stop accuracy, were calculated separately for all cue conditions. The SSRT was estimated using the integration method (Verbruggen et al., 2013), with replacement of go omissions, as suggested in a recent consensus paper regarding the stop-signal task (Verbruggen et al., 2019). Specifically, go omission trials (i.e., go-trials without a response) was replaced with the maximum go-RT for the specific cue-condition. Thus, the go-trial distribution included valid go-trials, erroneous go-trials (i.e., trials with a choice error) and the go omission replacement trials. The full go-RT distribution were rank ordered, and the $n$th RT was selected by determining $n$ as the product of multiplying the probability of responding in stoptrials with the number of all go-trials. Finally, the SSRT was estimated by subtracting the mean SSD from the $n$th RT. 
Not all data sets of behavioural performance measures were normally distributed. The behavioural measures related to go-trials (i.e., go-RT and go accuracy) were analysed using a one-way repeated measures ANOVA, which has been found to be quite robust against deviations from normality (Schmider et al., 2010). All measures related to stop-trials (i.e., SSRT, US-RT, SSD and stop accuracy) were compared using Wilcoxon Signed rank tests for dependent samples. All behavioural data were analysed in IBM SPSS Statistics for Windows, Version 25.0 (IBM Corp.).

\subsection{2 fMRI data: pre-processing. All pre-processing steps were performed with SPM12} (Wellcome Trust Centre for NeuroImaging, Institute of Neurology at University College London, UK), running under MATLAB r2016a (The MathWorks Inc., Natick, MA). Functional images were realigned to the first image and resliced using a $5^{\text {th }}$ degree B-spline interpolation. Slice timing correction to adjust for time acquisition delays was performed using the central slice as reference. Functional images were co-registered to the structural image, and normalised to the Montreal Neurological Institute (MNI) standard space, using linear and non-linear deformations, written with a final resolution of 3x3x3 mm. Lastly, functional images were smoothed using a $6 \mathrm{~mm}$ full-width-at-half-maximum (FWHM) Gaussian kernel. The estimated motion parameters were inspected to ensure that relative motion (between adjacent time points) did not exceed half the voxel size.

\subsection{2 fMRI data: analysis. First-level statistical analyses were performed in SPM12 in an} event-related design using the general linear model (GLM) framework. The following events were included in the model: cues, successful go-trials, erroneous go-trials, successful stoptrials, unsuccessful stop-trials, null-events and feedback. All task conditions were modelled according to their corresponding stop-signal probability. Go- and stop-trials were modelled at go-signal onset and stop-signal onset, respectively, while unsuccessful stop-trials were modelled at response onset. In addition, six regressors coding the participant's motion parameters estimated during the realignment procedure were included to correct for head movement. Task events were modelled as zero-duration events, with the exception of feedback, which was modelled with a 20 -second duration. All modelled events were convolved with a canonical hemodynamic response function (HRF). To account for slowfrequency drifts, a high-pass filter with cut-off at 128 seconds was implemented. 
The conditions of interest were the successful stop-trials in the $25 \%$ condition and the $66 \%$ condition (hereby referred to as stop25 and stop66), and the successful go-trials in the $0 \%, 25 \%$ and $66 \%$ condition (hereby referred to as go0, go25, and go66). To explore the global activity evoked during reactive inhibition, a stop > go t-contrast was created for each participant; i.e., the collapsed activity in the stop25 and stop66 conditions were compared against the collapsed activity in the go25 and go66 condition (hereby referred to as global whole-brain analysis). The resulting t-maps from all participants were then subjected to a group-level random effects analysis, where a one-sample t-test was used on the sample contrast images. To further explore the activity evoked during reactive inhibition, this procedure was also performed separately for a stop $25>$ go 25 contrast and for a stop66 > go66 contrast. As the main aim of the study was the investigation of activity within specific ROIs, the global whole-brain analysis was performed as a validity check of the paradigm. Thus, resulting t-value maps were analysed using the potentially more lenient cluster-level inference, with a height threshold of $p<.001$, and cluster probability of $p<.05$ (family wise error corrected; FWE).

ROIs were defined using the MarsBaR ROI toolbox version 0.44 for SPM (Brett et al., 2002). Nine ROIs were defined as spheres with a six-millimetre radius centred at coordinates of local maxima extracted from a meta-analysis conducted by Cieslik et al. (2015; see Table 1 and Figure 2). This meta-analysis was chosen because all studies included were studies using the standard stop-signal task, and coordinates were inferred from the contrast stop > go. We know of no more recent meta-analysis meeting these requirements. Furthermore, it included differential regions of the right inferior frontal area (i.e., the pars opercularis and the pars triangularis, as well as the right anterior insula). The regions used within the present study therefore represent a selection of those regions detected in the meta-analysis that is based on the specified a priori hypotheses. Thus, not all regions of the meta-analysis were included in the present study. For each participant, mean beta values were extracted from each ROI for the go0, go25, go66, stop25 and stop66 conditions. The extracted mean beta values were exported to IBM SPSS Statistics for Windows, Version 25.0 (IBM Corp.) for statistical analysis.

Table 1: Peak Local Maxima Coordinates of ROIs

\section{Region}

Right anterior insula

\section{MNI Coordinates $(x, y, z)$}

$34,22,-4$ 
Right IFG (pars opercularis)

Right IFG (pars triangularis)

Left anterior insula

Pre-SMA

Right MFG

aMCC

Right IPC

Striatum
$50,12,28$

$56,20,4$

$-40,16,-4$

4, 16, 48

$36,2,54$

$6,30,32$

$62,-42,26$

$-4,12,-2$

Note. IFG = inferior frontal gyrus, pre-SMA = pre-supplementary motor area, $\mathrm{MFG}=$ middle frontal gyrus, aMCC $=$ anterior cingulate cortex, $\mathrm{IPC}=$ inferior parietal cortex.

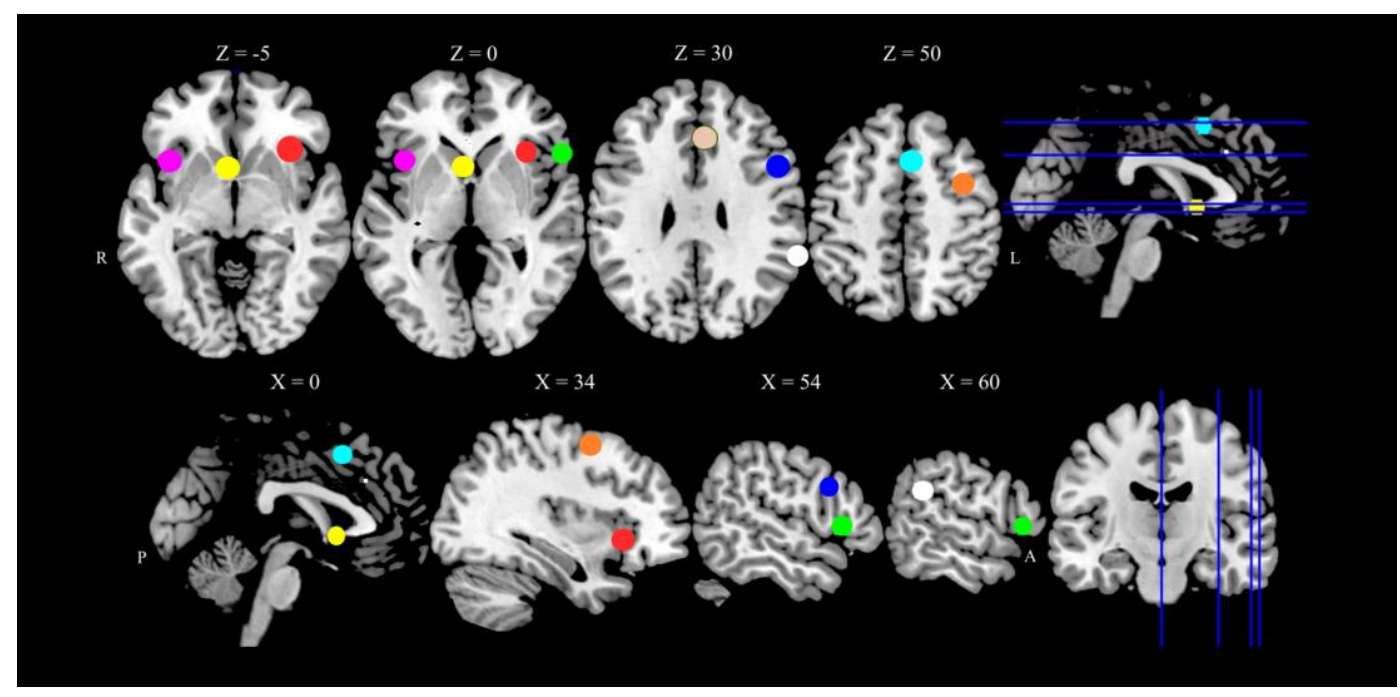

Figure 2: Graphical depiction of the ROI locations. Left insula in pink, striatum in yellow, right insula in red, pars triangularis in bright green, aMCC in beige, pars opercularis in dark blue, right IPC in white, pre-SMA in turquoise, right MFG in orange. $R=$ right, $L=$ left, $P=$ posterior, $A=$ anterior. Note: For accurate locations of the ROIs see Table 1. ROIs are projected onto the template ch2better of MRIcroN

(https://people.cas.sc.edu/rorden/mricron/index.html).

As the main interest of the present study was the effect of stop-signal probability on go-trial and stop-trial activity, rather than the difference between go and stop, the following ROI analyses were performed. To see how stop-signal probability affects go-trial brain activity, a 3 (stop-signal probability; 0, 25, 66) x 9 (ROI; left insula, pre-SMA, aMCC, right 
insula, right IPC, right MFG, pars opercularis, pars triangularis, striatum) repeated-measures ANOVA was performed on the corresponding beta values. To see how stop-signal probability affected the brain activity in the stop-trials, a 2 (stop-signal probability; 25, 66) x 9 (ROI; left insula, pre-SMA, aMCC, right insula, right IPC, right MFG, pars opercularis, pars triangularis, striatum) repeated measures ANOVA was set up.

In contrast to the previous analyses, which focused on the effects of proactive inhibitory control on specific regions of interest, two whole-brain analyses were performed with SSRT as covariate of interest (one for each stop-signal probability), to investigate the relationship between the behavioural performance of reactive inhibitory control and brain activity. That is, for the $25 \%$ condition, first level contrast images of stop $25>$ go 25 from all participants were subjected to a random effects multiple regression analysis with the SSRT in the $25 \%$ condition from each participant as a covariate of interest. This was then repeated for the $66 \%$ condition. Resulting t-value maps were analysed using voxel-wise inference with $\mathrm{p}<$ .05 (FWE). Similarly, to investigate which cortical regions might correlate with the behavioural indices of proactive inhibitory control induced by increased stop-signal probability, three difference scores were calculated: between go-RT in the go25 and the go0 condition (go-RT25 - go-RT0), as was the difference between go-RT in the go66 and go0 condition (go-RT66 - go-RT0), and in the go66 and go25 condition (go-RT66 - go-RT25). These estimated differences were then subjected as covariates of interest to three random effects multiple regression analyses, using first level contrast images of go25 > go0, go66 > go0 and go66 > go25, respectively. Group-level t-value maps were analysed using voxel-wise inference with $\mathrm{p}<.05$ (FWE).

Because of the specific hypotheses regarding the relationships of the pre-selected ROIs with both proactive and reactive inhibitory control, bivariate two-tailed correlations between the SSRT and ROI beta values for the $25 \%$ and $66 \%$ conditions, and one-tailed bivariate correlations between the go-RT difference estimates and the corresponding differences between ROI beta values were computed. The alpha level was Bonferroni-corrected for number of tests $(\alpha=.05 /(9 \times 2)=.0028)$. For all statistics, Greenhouse-Geisser corrected estimates are reported when the assumption of sphericity was violated.

Lastly, an exploratory psycho-physiological interaction (PPI) analysis was performed. PPI is a method that aims to determine which regions in the brain change their connectivity with a seed region when manipulating task context (Friston et al., 1997; O'Reilly et al., 2012). Three first level analyses were set up using the SPM toolbox for MATLAB. For all analyses, 
the rIFG pars opercularis (center: 50, 12, 28; radius: $6 \mathrm{~mm}$ ) was chosen as the seed region. Although there are some indications that specifying the region individually for each subject within some constrained area is the most sensitive approach (e.g., O'Reilly et al., 2012), the seed region was kept constant for all subjects for better correspondence with the ROI analysis. First, the region's time series was extracted for each subject, which represented the eigenvariate of the pre-whitened, high-pass filtered time series, adjusted for effects of interest. The PPI analysis was then conducted by using the predetermined region of interest and the contrast go66 > go25. The resulting PPI interaction term, the eigenvariate time series and the experimental task vector was then subjected to a GLM analysis, and a t-contrast image for the interaction term was computed. The resulting t-maps of all participants were then subjected to a group-level random effects analysis, where a one-sample t-test was used on the sample of contrast images. These steps were repeated for the contrast stop66 > stop25, and go66 > go0. The resulting whole-brain t-value maps were analyzed using voxel-wise inference with $\mathrm{p}<$ .05 (FWE).

\section{Results}

\subsection{Behavioural results.}

A summary of the behavioural measures is presented in Table 2. Overall, the participants performed well in all three cue-conditions, as evidenced by high go-trial accuracies of $95.7 \%$, $97.9 \%$ and $97.5 \%$ for the $0 \%, 25 \%$ and $66 \%$ conditions, respectively. There was a significant effect of stop-signal probability on go-trial accuracies $(F(1.33,35.81)=6.62, p=.009$, $\eta_{\mathrm{p}}{ }^{2}=0.20$ ), caused by a significantly higher accuracy in the $25 \%$ condition than in the $0 \%$ condition $(p=.006)$. The difference between the $25 \%$ and the $66 \%$ condition did not reach significance, nor did the difference between $0 \%$ and $66 \%(p>.05)$. There was also a significant effect of stop-signal probability on go-RT $(F(1.32,35.61)=104.74, p<.001$, $\left.\eta_{\mathrm{p}}{ }^{2}=0.80\right)$. Bonferroni-corrected post-hoc tests revealed that go-RT increased as a function of stop-signal probability. The go-RT in the $25 \%$ condition was significantly higher than in the $0 \%$ condition and in the $66 \%$ condition as compared the $25 \%$ condition (all $p<.001$ ).

The participants' accuracy in the stop-trials was close to 50\%, although the accuracy was higher in the $66 \%$ condition than in the $25 \%$ condition $(z=4.14, p<.001)$. US-RT increased with increasing stop-signal probability $(z=4.60, p<.001)$. Further, in line with 
assumption of the horse race model, the US-RT was significantly shorter than the go-RT in both the $25 \%$ condition $(z=-4.62, p<.001)$, and in the $66 \%$ condition $(z=-4.62, p<.001)$. Lastly, the SSD increased significantly with increasing stop-signal probability $(z=4.62, p<$ $.001)$. The difference between the SSRT in the $25 \%$ and in the $66 \%$ condition did not reach significance $(z=-1.62, p=.106)$.

Table 2. Behavioural data

\begin{tabular}{llll}
\hline & \multicolumn{3}{c}{ Stop-signal probability condition } \\
\hline & $\mathbf{0} \%$ & $\mathbf{2 5} \%$ & $\mathbf{6 6} \%$ \\
\hline Go-trial RT (ms)** & $438.58(57.44)$ & $592.73(64.74)$ & $685.15(115.44)$ \\
Go-trial accuracy (\%)* & $95.71(4.38)$ & $97.89(2.68)$ & $97.49(2.70)$ \\
SSRT (ms) & - & $250.91(53.45)$ & $227.29(51.07)$ \\
SSD (ms)** & - & $337.21(89.34)$ & $435.83(112.03)$ \\
US-RT (ms)** & - & $524.04(56.14)$ & $598.14(80.57)$ \\
Stop-trial accuracy $(\%)^{* *}$ & - & $48.86(6.07)$ & $52.53(5.36)$
\end{tabular}

Note. Table shows means of behavioural measures for each probability condition. Standard deviations are presented in the parentheses. $\mathrm{RT}=$ reaction time, $\mathrm{SSRT}=$ stop-signal reaction time, SSD = stop-signal delay, US-RT = unsuccessful stop-trial reaction time, $\mathrm{ms}=$ milliseconds. Significant differences marked with $* *(p<.001)$ and $*(p<.05)$.

\subsection{Imaging results.}

\subsubsection{Whole-brain.}

Global stop vs go effects in brain activity. Contrasting stop > go, cluster-level inference revealed significant activity in bilateral IFG pars orbitalis and bilateral MFG (all $p<.05$ ), which is in line with the previous literature (Aron \& Poldrack, 2006; Jahfari et al., 2010). However, the additional contrasts investigating stop-related brain activity in the $25 \%$ and $66 \%$ conditions separately seem to suggest differences between the two conditions, although no direct comparisons was performed on whole-brain data. See Table 3 for all significantly activated clusters, visually displayed in Figure 3. 


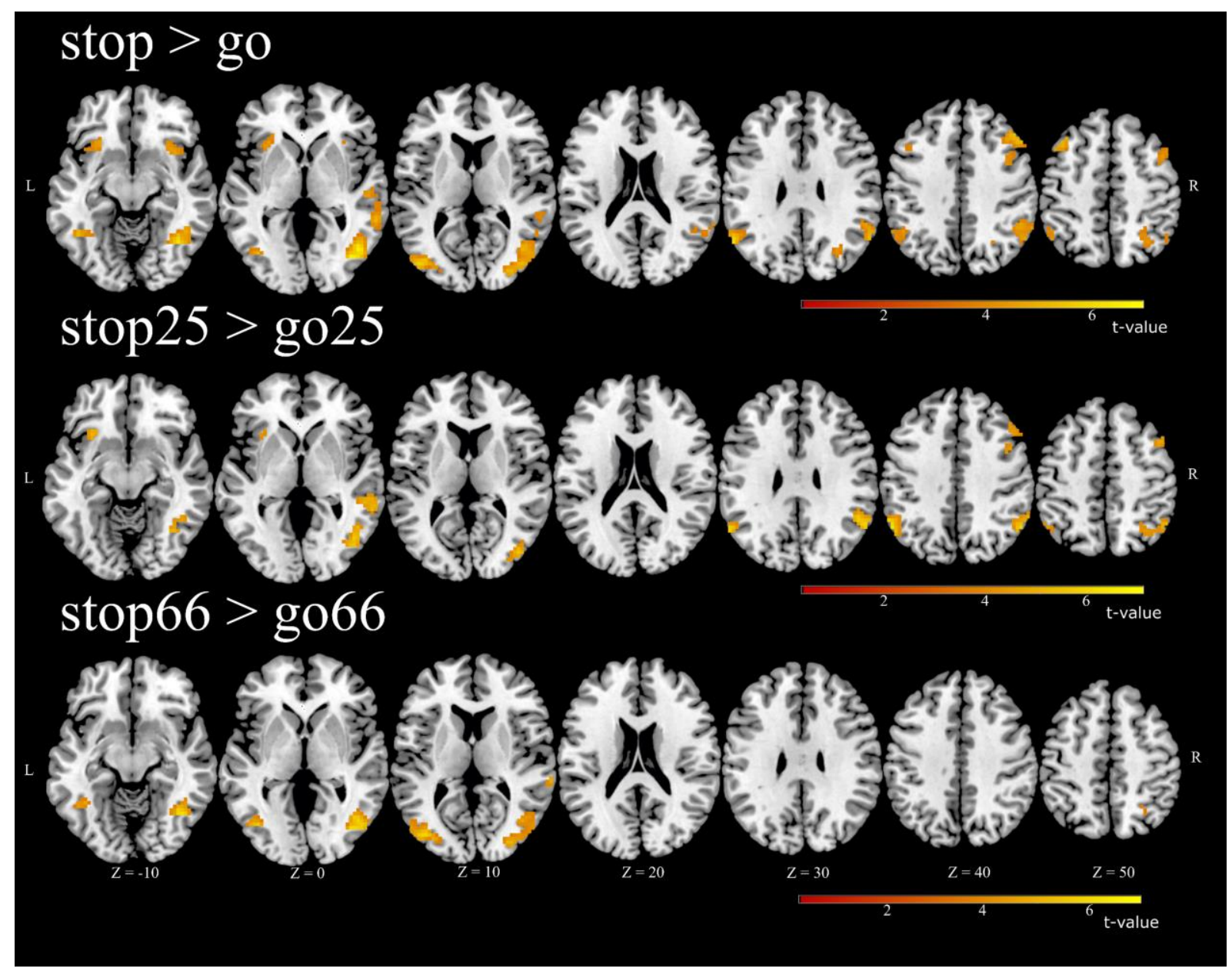

Figure 3: Whole brain analysis overlaid on single-subject template brain. Height threshold of $p<.001$, cluster probability of $p<.05(F W E)$. Colour scale in $t$-value units. $R=$ right, $L=$ left. Activation maps were projected onto the template ch2better of MRIcroN (https://people.cas.sc.edu/rorden/mricron/index.html).

Table 3. Activated clusters contrasting stop $>$ go.

\begin{tabular}{lccccc}
\cline { 1 - 4 } stop > go. & & & & & Cluster \\
Region & X (mm) & Y (mm) & Z (mm) & T & extent \\
\hline L Middle Occipital Gyrus & -42 & -79 & 8 & 6.99 & 141 \\
R Middle Occipital Gyrus & 45 & -79 & 5 & 6.79 & 687 \\
L Angular Gyrus & -57 & -61 & 29 & 6.02 & 120 \\
R Middle Frontal Gyrus & 45 & 29 & 44 & 5.36 & 118 \\
L Middle Frontal Gyrus & -39 & 20 & 50 & 4.88 & 46 \\
R IFG (p. orbitalis) & 36 & 17 & -19 & 4.70 & 58 \\
L IFG (p. orbitalis) & -30 & 23 & -16 & 4.47 & 52
\end{tabular}




\begin{tabular}{|c|c|c|c|c|c|}
\hline $\begin{array}{l}\text { R Angular Gyrus } \\
\text { stop25 > go } 25\end{array}$ & 30 & -58 & 53 & 4.43 & 90 \\
\hline Region & $\mathrm{X}(\mathrm{mm})$ & $\mathrm{Y}(\mathrm{mm})$ & $\mathrm{Z}(\mathrm{mm})$ & $\mathrm{T}$ & $\begin{array}{c}\text { Cluster } \\
\text { extent }\end{array}$ \\
\hline L Inferior Parietal Lobule & -57 & -55 & 38 & 6.49 & 113 \\
\hline R Angular Gyrus & 51 & -55 & 35 & 5.96 & 201 \\
\hline $\begin{array}{l}\text { R Middle Occipital Gyrus } \\
\text { R Inferior Temporal }\end{array}$ & 45 & -79 & 5 & 5.60 & 140 \\
\hline $\begin{array}{l}\text { Gyrus } \\
\text { R Middle Temporal }\end{array}$ & 45 & -49 & -13 & 5.48 & 62 \\
\hline Gyrus & 54 & -40 & 5 & 4.85 & 85 \\
\hline L Insula Lobe & -30 & 20 & -7 & 4.74 & 51 \\
\hline R Middle Frontal Gyrus & 42 & 11 & 44 & 4.68 & 66 \\
\hline
\end{tabular}

stop66 > go66

\begin{tabular}{|c|c|c|c|c|c|}
\hline Region & $\mathrm{X}(\mathrm{mm})$ & $\mathrm{Y}(\mathrm{mm})$ & $\mathrm{Z}(\mathrm{mm})$ & $\mathrm{T}$ & $\begin{array}{c}\text { Cluster } \\
\text { extent }\end{array}$ \\
\hline L Middle Occipital Gyrus & -45 & -79 & 8 & 7.04 & 175 \\
\hline R Middle Temporal Gyrus & 48 & -73 & 2 & 6.34 & 342 \\
\hline R Superior Parietal Lobe & 33 & -49 & 56 & 4.92 & 48 \\
\hline
\end{tabular}

Note. MNI coordinates. Anatomical labelling by the SPM Anatomy toolbox (Eickhoff et al., 2005). Height threshold of $\mathrm{p}<.001$, cluster probability of $\mathrm{p}<.05$ (FWE). $\mathrm{R}=$ right, $\mathrm{L}=$ left. $\mathrm{IFG}=$ Inferior frontal gyrus. $\mathrm{X}, \mathrm{Y}, \mathrm{Z}$ coordinates listed are the location of the peak $\mathrm{T}$ value in each cluster.

\subsubsection{ROI analyses.}

Go-related changes in brain activity. To investigate the effect of proactive control on go-trial activity, a 3 (stop-signal probability; 0\%, 25\%, 66\%) x 9 (ROI; left insula, pre-SMA, aMCC, right insula, right IPC, right MFG, pars opercularis, pars triangularis, striatum) repeatedmeasures ANOVA was performed with go-related beta values as the dependent variable. The ANOVA revealed a significant main effect of stop-signal probability $(F(2,54)=5.45, p=$ $\left..007, \eta_{\mathrm{p}}^{2}=0.17\right)$, and $\operatorname{ROI}\left(F(8,216)=14.32, p<.001, \eta_{\mathrm{p}}{ }^{2}=0.35\right)$, as well as a significant interaction between stop-signal probability and $\operatorname{ROI}(F(7.67,207.19)=4.07, p<.001$, $\left.\eta_{\mathrm{p}}{ }^{2}=0.13\right)$. As evident from Figure 4A, Bonferroni-corrected post-hoc tests revealed that the bilateral insula, the pars opercularis, and the pars triangularis all showed a significant increase in activity from the $25 \%$ to the $66 \%$ condition (all $p<.05$, Bonferroni corrected).

Furthermore, left insula, right IPC, right MFG, and the pars triangularis all exhibited a 
significant decrease in activity from the $0 \%$ to the $25 \%(p<.05$, Bonferroni corrected). The right insula was the only region showing higher activity in the $66 \%$ condition than in the $0 \%$ condition ( $p=.002$, Bonferroni corrected). Interestingly, the pre-SMA, aMCC and striatum did not show these probability-specific modulations.

Stop-related changes in brain activity. To investigate the effect of proactive inhibitory control and its interaction with the reactive stop-trial activity, a 2 (stop-signal probability; $25 \%, 66 \%)$ x 9 (ROI; left insula, pre-SMA, aMCC, right insula, right IPC, right MFG, pars opercularis, pars triangularis, striatum) repeated measures ANOVA was performed. The interaction effect between stop-signal probability and $R O I$ was significant $(F(8,216)=2.78$, $\left.p=.006, \eta_{\mathrm{p}}^{2}=0.09\right)$, as were the main effects of both stop-signal probability $(F(1,27)=$ $\left.8.11, p=.008, \eta_{\mathrm{p}}^{2}=0.23\right)$, and $\operatorname{ROI}\left(F(5.46,147.50)=15.64, p<.001, \eta_{\mathrm{p}}{ }^{2}=0.37\right)$. The main effect of stop-signal probability indicated generally higher activity in the $66 \%$ condition than the $25 \%$ condition. However, with respect to the interaction of stop-signal probability and ROI, follow-up Bonferroni-corrected post-hoc tests revealed some regional specificity with higher activity in the $66 \%$ condition than in the $25 \%$ condition in the bilateral insula, preSMA, aMCC, and the pars opercularis, while no significant differences between conditions were found for the right IPC, right MFG, the pars triangularis, and the striatum (Figure 4B). 


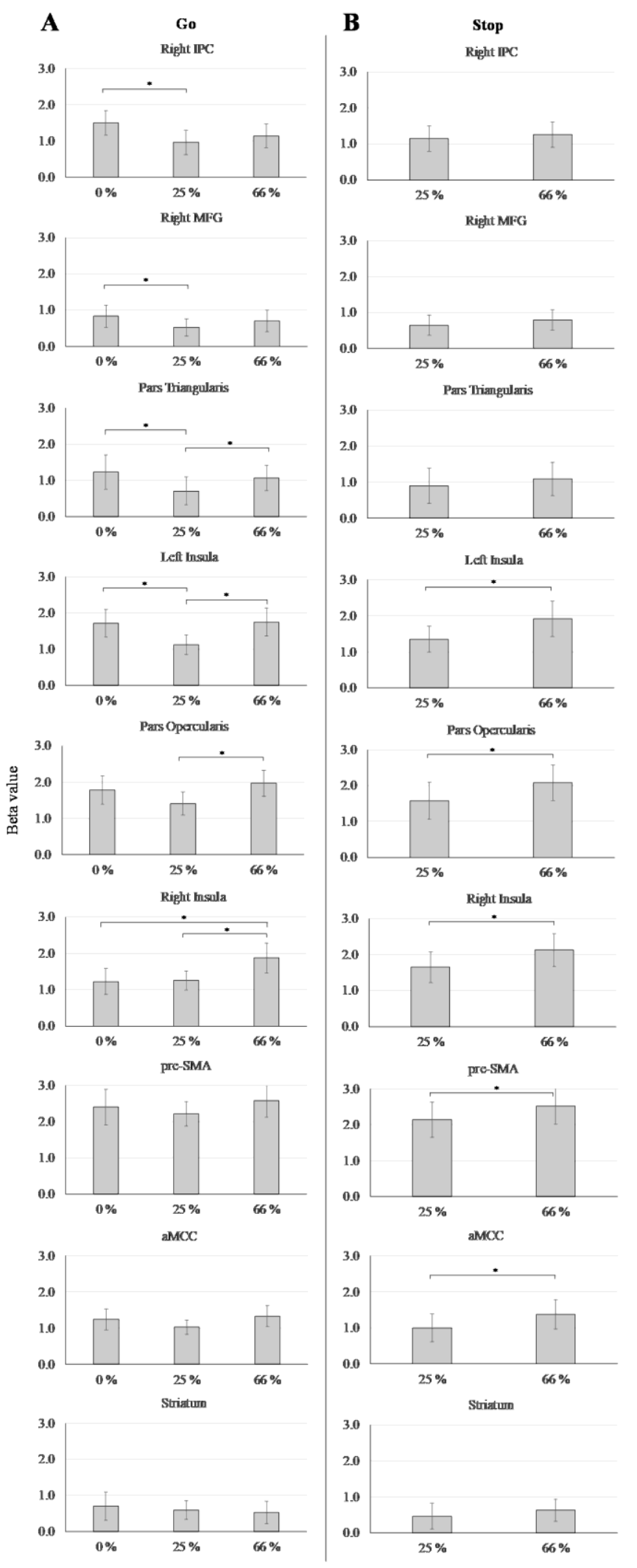

Figure 4: Effect of stop-signal probability on A) go-trial activity and B) stop-trial activity. Figure shows Bonferroni corrected pairwise comparisons of the interaction effect between ROI and stop-signal probability. Significant differences are marked with $*(p<.05$, Bonferroni corrected). Error bars represent $95 \%$ confidence intervals. aMCC = anterior midcingulate cortex, pre-SMA = presupplementary motor area, $I P C=$ inferior parietal cortex, $M F G=$ middle frontal gyrus.

\subsubsection{Brain-behaviour correlations.} Whole brain analyses testing for correlations between the SSRTs and stop-trial related brain activity revealed a significant negative correlation between the right thalamus and SSRT in the $66 \%$ condition, although the cluster was quite small (Table 4). No significant activity was evident for the $25 \%$ condition.

Two-tailed bivariate correlations between SSRTs and ROI stop-trial beta values for both the $25 \%$ and the $66 \%$ condition did not reach

significance (all $p>.0056)$.

The whole-brain analysis investigating whether there was a relationship between the increase in go-RT and an increase in brain activity between the different stop-signal 
probability conditions did not reveal significant activations for the increase from $0 \%$ to $25 \%$, $0 \%$ to $66 \%$ nor from $25 \%$ to $66 \%$.

Table 4. Negative correlation between SSRT and whole brain activity

\begin{tabular}{llllll}
\hline Region & $\mathrm{X}(\mathrm{mm})$ & $\mathrm{Y}(\mathrm{mm})$ & $\mathrm{Z}(\mathrm{mm})$ & $\mathrm{T}$ & Cluster extent \\
\hline R Thalamus & 9 & -19 & 17 & 6.68 & 1
\end{tabular}

Note. Results of the whole brain analyses testing for correlations between the SSRTs and stop-trial related brain activity in the $66 \%$ condition. MNI coordinates. Anatomical labelling by the SPM Anatomy toolbox (Eickhoff et al., 2005). Voxel-wise inference with $p<.05$ (FWE). $\mathrm{R}=$ right, $\mathrm{L}=$ left. $\mathrm{X}, \mathrm{Y}, \mathrm{Z}$ coordinates listed are the location of the peak $\mathrm{T}$ value in each cluster.

There was a significant bivariate correlation between the difference of ROI beta values in the $66 \%$ and $25 \%$ conditions and the corresponding difference in go-RT, with stronger increase in activity being associated with more pronounced RT slowing (Figure 5). This was true for the left insula $(r=0.517, p=.002)$, aMCC $(r=0.534, p=.002)$, right insula $(r=$ $0.532, p=.002)$, and the pars triangularis $(r=0.624, p<.001)$. The pre-SMA exhibited a relationship of similar strength $(r=0.468, p=.006$ ), that nevertheless did not cross the Bonferroni corrected significance level. No significant bivariate correlations were found for the differencesof go66 > go0 or go25> go0. 

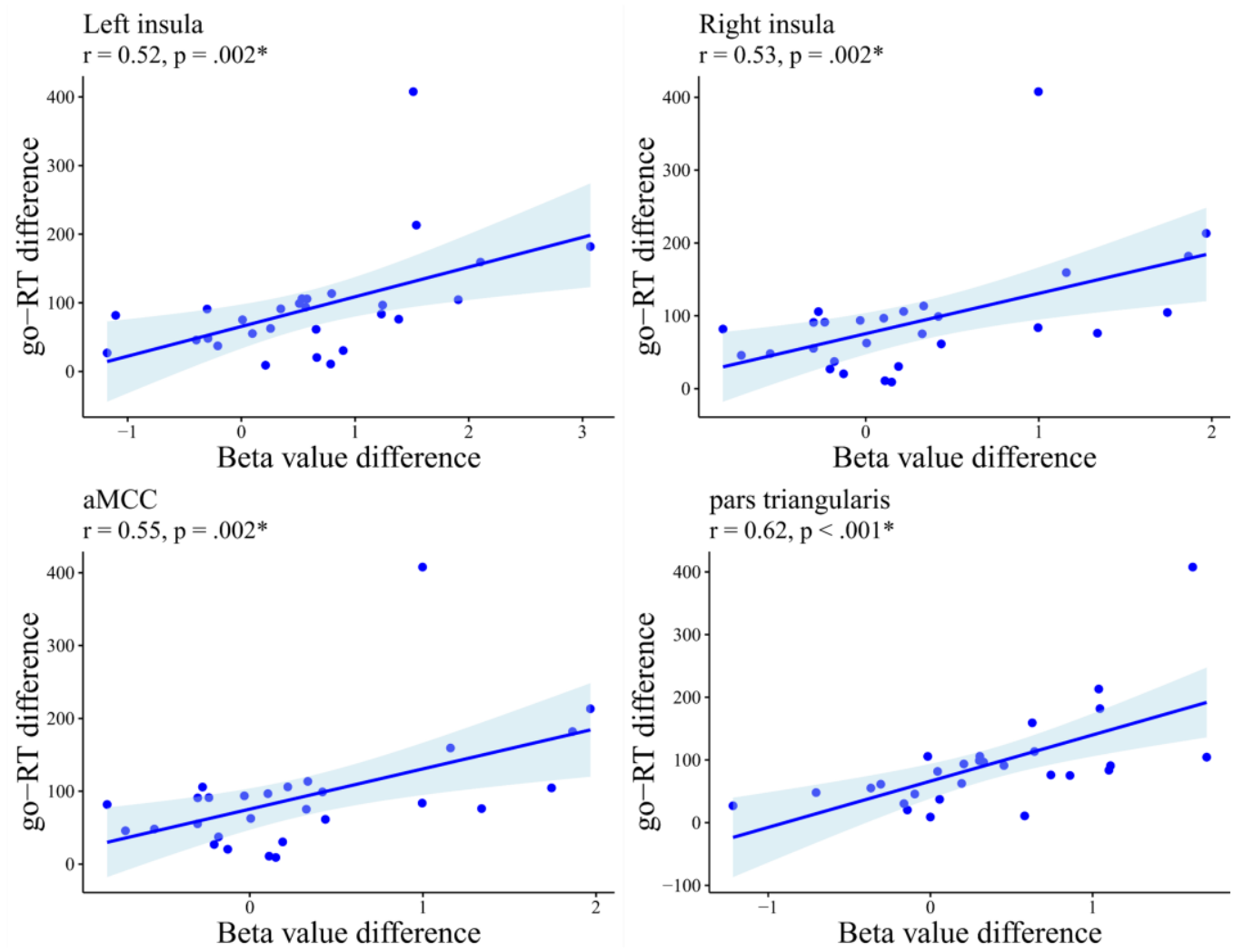

Figure 5: Bivariate one-tailed correlations revealed a significant correlation between go-RT difference and ROI beta value difference between the $66 \%$ and $25 \%$ conditions. Significant effects are marked with * $(p<.0028)$.

\subsubsection{PPI analysis.}

PPI analyses of the go-trials revealed a significant increase in connectivity between the pars opercularis and the left putamen as well as the right fusiform gyrus in the $66 \%$ condition relative to the $25 \%$ condition (Table 5). However, the significant clusters were rather small. Neither the corresponding effects for stop-signal trials, nor the go66 > go0 analysis revealed any significant changes in connectivity.

Table 5. PPI analysis.

\begin{tabular}{llllll}
\hline Go66 > go25 & & & & & \\
\cline { 1 - 4 } Region & $\mathrm{X}(\mathrm{mm})$ & $\mathrm{Y}(\mathrm{mm})$ & $\mathrm{Z}(\mathrm{mm})$ & $\mathrm{T}$ & Cluster extent \\
\hline L Putamen & -12 & 11 & -7 & 6.42 & 1 \\
R Fusiform Gyrus & 39 & -43 & -19 & 6.07 & 1 \\
\hline
\end{tabular}


Note. Results of the PPI analysis using the rIFG pars opercularis as seed region. MNI coordinates. Anatomical labelling by the SPM Anatomy toolbox (Eickhoff et al., 2005). Voxel-wise inference with $\mathrm{p}<.05$ (FWE). $\mathrm{R}=$ right, $\mathrm{L}=$ left. $\mathrm{X}, \mathrm{Y}, \mathrm{Z}$ coordinates listed are the location of the peak $\mathrm{T}$ value in each cluster.

\section{Discussion}

A cued stop-signal task was used to investigate the effects of proactive inhibitory control on response initiation and inhibition processes. As was expected based on previous research, whole-brain analyses revealed that inhibitory control was associated with activity in the bilateral IFG, MFG and IPC (e.g., Aron \& Poldrack, 2006; Chikazoe et al., 2009). More importantly though, behavioural and imaging results indicate that the participants adjusted their performance proactively with increasing stop-signal probability. That is, go-RT and stop-trial accuracy increased with increasing stop-signal probability, while the SSRT remained unaltered. Thus, participants used the probability cues to switch between task strategies, from a go-oriented mode with short response times, to a stopping mode with slow responses but higher stop-trial accuracy. The behavioural effects of mode switches were also reflected in the imaging data, where regions traditionally involved in outright stopping, such as the pre-SMA, pars opercularis, and the insula all showed stronger stop-trial activations in the $66 \%$ condition than in the $25 \%$ condition. Interestingly, go-trial activity was significantly higher in the $0 \%$ condition than the $25 \%$ condition in the left insula, pars triangularis, right MFG and right IPC. However, while the left insula and pars triangularis showed a significant increase in go-trial activity from the $25 \%$ to the $66 \%$ condition, this was not true for the MFG and IPC. This indicates that the mode-switching evident in the behavioural data is associated with different regions of the stopping network, with some regions being more associated with the facilitation of fast responses in the $0 \%$ condition, and other regions with efficient inhibition in the $66 \%$ condition. Furthermore, the results indicate that sub-regions in the inferior frontal area exhibit different activity patterns in response to proactive inhibitory control modulations, with the pars opercularis and right insula being more responsive to outright stopping, and the pars triangularis possibly being more associated with go-related processes during clear stimulus-response mappings.

\subsection{Mode-switching of task-strategies}


The increase in go-RT with increasing stop-signal probability is in line with previous research (Albares et al., 2014; Zandbelt \& Vink, 2010), and has been attributed to an increase in proactive inhibitory control (e.g., Verbruggen \& Logan, 2009b). Although it was expected that the SSRT would decrease with increasing stop-signal probability as a result of the increase in proactive inhibitory control, no such effect was found in the present study. The lack of a decrease in the SSRT has also been found in previous research (e.g., Lavallee et al., 2014; Leunissen et al., 2016), and has been attributed to a response-strategy adjustment where speed in the go-trials are traded for accuracy in the stop-trials (Verbruggen and Logan, 2009b). This was supported by the stop-trial accuracy, which was significantly higher in the $66 \%$ condition than in the $25 \%$ condition. Thus, the behavioural data indicate a shift from a predominately "go-mode" in the $0 \%$ condition to a predominately "stop-mode" in the $66 \%$ condition. Furthermore, the behavioural pattern observed in the $25 \%$ condition clearly indicates that participants tried to balance response speed and stopping accuracy (Verbruggen $\&$ Logan, 2008). In the $0 \%$ condition, participants do not have to proactively prepare for upcoming inhibition, and this certainty of a motor execution facilitates processes supporting fast responses. The added uncertainty in the $25 \%$ condition urges participants to apply a more balanced strategy, as evident in higher go-RTs compared to the $0 \%$ condition. In the $66 \%$ condition, participants clearly engage in efficient stopping. This is indicated by increased stopping accuracy, coming at the expense of prolonged go-RTs. This pattern further supports previous research that has found that people switch task strategies in accordance with the frequency and relevance of stop-trials (Bissett \& Logan, 2014).

\subsubsection{Regions facilitating fast responding in go-trials}

The mode-switching of task-strategies evident in the behavioural data is mirrored in the neural data. That is, the facilitation of fast responses in the $0 \%$ condition and the facilitation of efficient stopping in the $66 \%$ condition show associations with distinct regions of the stopping network. The right IPC, right MFG, pars triangularis and the left insula all showed a significantly increased go-trial activity in the $0 \%$ condition compared to the $25 \%$ condition. It is somewhat surprising though that certain regions of the stopping network showed this elevated go-trial activity in the $0 \%$ condition compared to the $25 \%$ condition. Although speculative, this effect may reflect the certainty of an upcoming motor response and the allocation of attentional resources to the detection of the go-signal. That is, during conditions when the need for a response to the go-signal is certain (i.e., the $0 \%$ condition), attentional 
processes allocated to the detection of the target as well as facilitation of the fast response are recruited. A recent attempt to investigate the interaction between attentional resources and response inhibition did indeed find a down-modulation of go-stimulus processing in conditions when the probability of stopping is high, reflected in a relationship between attenuated EEG N1 amplitudes (a marker for early visual-sensory processing) and go-RTs (Langford et al., 2016). The opposite pattern (i.e., up-modulation of go-stimulus processing in conditions of low proactive inhibitory control) thus seems plausible. Since the N1 amplitude decreased with go-RT slowing, it is likely that these processes are under proactive control (Langford et al., 2016). As the rIPC has been associated with attentional processes during stop-signal tasks (Gur et al., 2007; Hampshire et al., 2010; Shulman et al., 2009; Zandbelt et al., 2013), an effect that seems to be independent of the task-relevance of the stop-signal (Boehler et al., 2011), it may be that the attentional processes related to the N1 modulation indeed are related to IPC activity. The reported associations of the IPC with both the maintenance of attentional control (Singh-Curry \& Husain, 2009) and the bottom-up detection of salient stimuli (Corbetta \& Shulman, 2002) further support this interpretation.

Both the pars triangularis and the left insula showed a more task-general pattern, with higher go-trial activity both in the $0 \%$ condition and in the $66 \%$ condition, than in the $25 \%$ condition. Thus, it may be that the activity in these regions is associated with general processes induced by the clear stimulus-response mappings of these two conditions. That is, during the $0 \%$ condition, these regions may help to facilitate the fast responding as evident by the short go-RTs. In the $66 \%$ condition however, the activity in these regions may be associated with a general response slowing, as a result of the increased proactive inhibitory control induced by the increased stop-signal probability. This is supported by the correlation between the behavioural slowing from the $25 \%$ condition to the $66 \%$ condition and the activity difference between these conditions in both the pars triangularis and the left insula.

\subsubsection{Mechanisms of efficient stopping during proactive inhibitory control}

The bilateral insula, pars opercularis, pre-SMA and aMCC all showed a significant increase in stop-trial activity from the $25 \%$ condition to the $66 \%$ condition. Thus, during increased proactive control there is an increase in activity in regions traditionally involved in reactive inhibitory control. This indicates that there is an interaction between the processes governing the two modes of inhibitory control. 
Interestingly, for the pre-SMA and the aMCC, the increase in activity from the $25 \%$ condition to the $66 \%$ condition was not evident in go-trial activity. This may indicate that the inhibition-related processes associated with pre-SMA activity are occurring after, or related to, the detection of the stop-signal, such as connectivity with the basal ganglia to modulate response or inhibition thresholds. In fact, pre-SMA connections to the basal ganglia have been found to be modulated by stop-signal probability and trial outcome certainty (Forstmann et al., 2010; Jahfari et al., 2012). Although no significant difference between aMCC go-trial activity in the $25 \%$ and the $66 \%$ condition was found, this difference was nevertheless correlated with behavioural adaptions in the go-RT between these two probability conditions. The aMCC has also been implicated in response preparation and working memory (reviewed in Vogt, 2016). Thus, it may be that the activity pattern seen here indicates that the aMCC is involved in processes related to proactive inhibitory control, and processes beyond those introduced by the stop-signal. Future studies need to investigate this further.

\subsection{Dissociable functional roles of inferior frontal sub-regions}

Despite the association of the (right) IFG with response inhibition, its exact functional role has become the center of discussion. It has been argued that the exact choice of IFG-ROIs may greatly alter the observed response profile to task challenges (e.g., Aron, 2011; Hampshire et al., 2010). Indeed, our results indicate such a functional dissociation of the right inferior frontal area. The pars opercularis and the right insula showed the expected stoppingactivity, with higher activity in the $66 \%$ condition than in the $25 \%$ condition, in both go- and stop-trials. This is consistent with the idea that the rIFG acts as a brake during conditions of high proactive inhibitory control (Aron et al., 2014). Here, we show that this braking mechanism is not specific to the pars opercularis, but that the right insula also exhibits a compatible response profile. It has been argued that the insula activity during stop-signal tasks rather reflects autonomic arousal related to stopping (Aron et al., 2014) or the detection of behaviorally salient events (Cai et al., 2014), instead of inhibition per se. Here, we show that insula activity is susceptible to proactive modulations of stopping, which may indicate more than pure autonomic arousal. If the insula activity was dependent on the saliency of the stopsignal, then a decrease in activity with increasing stop probability would be more likely reflecting the increased expectancy of the stop-signal. Furthermore, the activity difference from the $25 \%$ to the $66 \%$ condition in the right insula was correlated with the corresponding go-RT difference, a correlation not evident for the pars opercularis. These findings further 
support the notion that the insula's function extends beyond mere autonomic arousal or attentional mechanisms.

Interestingly, the pars triangularis showed a somewhat different activity pattern than the two other inferior frontal regions. While the pars triangularis did show a significant increase in activity from the $25 \%$ to the $66 \%$ condition, this was only true for go-trials. Furthermore, this was accompanied by a significant correlation with the behavioral RT difference between these two conditions. In the stop-trials, no significant modulations were evident in this region. Interestingly, the pars triangularis, as the only inferior frontal subregion, showed a significant decrease in go-trial activity from the $0 \%$ to the $25 \%$ condition. Thus, while the pars opercularis and right insula may be more susceptible to proactive modulations of outright stopping, the pars triangularis activity pattern rather indicates an association with conditions favoring task modes with clear stimulus-response mappings. The present results further support previous research showing that more posterior parts of the inferior frontal area are indeed related to stopping and inhibition (Hartwigsen et al., 2018).

The PPI analysis revealed that go-trials during conditions of high compared to low proactive inhibitory control exhibited significantly higher connectivity between the pars opercularis and the left putamen and right fusiform gyrus. No such connectivity patterns were evident in the stop-trials. These results support the idea of the pars opercularis being implicated in proactive inhibitory control, possibly as a braking mechanism (Aron et al., 2014), and suggest that this braking mechanism may involve the striatum of the basal ganglia. Further, the results of the PPI analysis opens up for a dissociation of the functional roles of the pars opercularis and the right insula, as no connectivity between these regions was evident. However, some important limitations of the PPI analyses need to be addressed. First, PPI analyses usually suffer from a lack of power, owing to the modeling of changes of connectivity as an interaction term to exploit the residual variance of other regressors (O’Reilly et al., 2012). Further, and perhaps as a consequence of low power and sensitivity, the significant clusters evident in go-trials were rather small. The PPI results thus await further replication, not least through the use of alternative methods for the detection of connectivity patterns.

\subsection{Future directions and limitations}


To our surprise, the expectation that activity in go-trials would increase concurrently with the augmentation of stopping probability from $0 \%$ to $25 \%$ in regions associated with proactive inhibitory control was not met. Since failures to find increases in go-trial activity from low to high stop-signal probability in regions such as the rIFG have been reported earlier (Jahfari et al., 2012; Leunissen et al., 2016), we believe that such outcomes are based in specifics of the task design. As the distance between the probabilities chosen for the current design is larger than what has been used in previous research varying stop-signal probabilities, it may have triggered more pronounced strategic adaptations in the $0 \%$ and the $66 \%$ condition, leaving the $25 \%$ condition to be the most uncertain condition. This suggests that specific care has to be taken when using stopping probabilities as experimental factors, and future research is needed to further dissociate strategic adaptations from genuine inhibitory and attentional processes.

The ROIs chosen in the present study was inferred form a meta-analysis using the standard stop-signal task, and the contrast stop > go (Cieslik et al., 2015). It is interesting then that regions traditionally involved in stopping, such as the striatum, do not show activity modulations as an effect of increased proactive inhibitory control. Although the present results may indicate that the striatum rather exhibits proactive modulations through connectivity changes, this notion needs to be cross-validated with alternative analysis schemes. The striatum ROI used here was located to the left caudate, and it may be that proactive inhibitory control recruits other regions of the basal ganglia. Leunissen et al. (2016) found an increased go-trial activation in right putamen during low compared to high stopsignal probability, indeed showing that different regions of the caudate may show different activity patterns in response to proactive inhibitory control modulations. Further, correlational analysis in the present study did reveal an association between the SSRT and the right thalamus that was only evident in the $66 \%$ condition. Although speculative considering the cluster size of this result, it does support the notion that proactive inhibitory control affects basal ganglia pathways (Aron, 2011; Leunissen et al., 2016). It may be that choosing a striatal ROI based on local maxima coordinates from research on proactive inhibitory control (as opposed to the standard SST) would have yielded different results, and future research should investigate this further.

It needs to be noted that the contributions of proactive and reactive inhibitory control to the net effects discussed here cannot be dissociated in this study because of the temporal proximity of the cue and the target stimulus. Thus, we assume that proactive inhibitory control increases with increasing stop-signal probability as we see a corresponding increase in 
go-RT. However, as we do not see the corresponding activity increase in the regions associated with proactive inhibitory control from the $0 \%$ to the $25 \%$ condition, we can only hypothesize that the different conditions trigger distinct strategy modes as reflected in different activity patterns. In addition, the exact neural mechanisms through which proactive inhibitory control exerts its influence, e.g., how proactive inhibitory control interacts with reactive inhibitory processes, is not known. Future studies should test the interaction between proactive and reactive inhibitory processes specifically.

\section{Conclusion}

The present study is the first to report how varying proactive inhibitory control results in the switching of task or strategy modes, either favouring fast responding or fast stopping, and how this process is governed by different regions of the stopping network. Furthermore, using a parametric design, as opposed to only binary comparisons of certain to uncertain trials, helped to elucidate the net effects of the interaction of proactive and reactive mechanisms on regional activity. Lastly, the present study suggests a functional dissociation of the right inferior frontal area, where the pars opercularis and right insula seem to be more associated with outright stopping, while the pars triangularis rather is associated with clear stimulusresponse mappings in go-trials.

Funding. This research did not receive any specific grant from funding agencies in the public, commercial, or not-for-profit sectors

Declarations of interest: none 


\section{References}

Albares, M., Lio, G., Criaud, M., Anton, J., Desmurget, M., \& Boulinguez, P. (2014). The Dorsal Medial Frontal Cortex Mediates Automatic Motor Inhibition in Uncertain Contexts: Evidence from Combined fMRI and EEG Studies. Human Brain Mapping, 35(11), 5517-5531. doi: 10.1002/hbm.22567

Aron, A. R. (2007). The Neural Basis of Inhibition in Cognitive Control. The Neuroscientist, 13(3), 214-228. doi: 10.1177/1073858407299288

Aron, A. R. (2011). From Reactive to Proactive and Selective Control: Developing a Richer Model for Stopping Inappropriate Responses. Biological Psychiatry, 69(12), e55-e68doi:10.1016/j.biopsych.2010.07.024

Aron, A.R., Behrens, T.E., Smith, S., Frank, M. J., \& Poldrack, R. A. (2007). Triangulating a Cognitive Control Network Using Diffusion-Weighted Magnetic Resonance Imaging (MRI) and Functional MRI. The Journal of Neuroscience, 27(14). 3743-3752. doi: 10.1523/JNEUROSCI.0519-07.2007

Aron, A. R., \& Poldrack, R. A. (2006). Cortical and Subcortical Contributions to Stop Signal Response Inhibition: Role of the Subthalamic Nucleus. The Journal of Neuroscience, 26(9), 2424-2433. doi: 10.1523/JNEUROSCI.4682-05.2006

Aron, A. R., Robbins, T. W., \& Poldrack, R. A. (2014). Inhibition and the right inferior frontal cortex: one decade on. Trends in Cognitive Sciences, 18(4), 177-185. doi:10.1016/j.tics.2013.12.003

Band, G. P. H., Molen, M. W., \& Logan, G. D. (2003). Horse-race model simulations of the stop-signal procedure. Acta Psychologica, 112. 105-142. Doi: 10.1016/S00016918(02)00079-3

Bissett, P. G., \& Logan, G. D. (2014). Selective stopping? Maybe not. Journal of Experimental Psychology: General, 143(1), 455-472. doi:10.1037/a0032122

Boehler, C. N., Appelbaum, L. G., Krebs, R. M., Chen, L., \& Woldorff, M. G. (2011). The Role of Stimulus Salience and Attentional Capture Across the Neural Hierarchy in a Stop-Signal Task. PLoS ONE, 6(10), e26386. Doi: 10.1371/journal.pone.0026386 
Botvinick, M. M., Cohen, J. D., \& Carter, C. S. (2004). Conflict monitoring and anterior Cingulate cortex: an update. TRENDs in Cognitive Sciences, 8(12). 539-546. doi:10.1016/j.tics.2004.10.003

Braver, T. S. (2012). The variable nature of cognitive control: A dual-mechanisms framework. Trends in Cognitive Sciences, 16(2), 106-113. doi:10.1016/j.tics.2011.12.010.

Brett, M., Anton, J., Valabregue, R., \& Poline, J. (2002). Region of interest analysis using an SPM toolbox [abstract]. Presented at the 8th International Conference on Functional Mapping of the Human Brain, June 2-6, 2002, Sendai, Japan. Available on CD-ROM in NeuroImage, 16(2).

Cai, W., Ryali, S., Chen, T., Li, C. R., \& Menon, V. (2014). Dissociable Roles of Right Inferior Frontal Cortex and Anterior Insula in Inhibitory Control: Evidence from Intrinsic and Task-Related Functional Parcellation, Connectivity, and Response Profile Analyses across Multiple Datasets. The Journal of Neuroscience, 34(44). 1465214667. doi:10.1523/JNEUROSCI.3048-14.2014

Chikazoe, J., Jimura, K., Hirose, K., Yamashito, K., Miyashita, Y., \& Konishi, S. (2009). Preparation to Inhibit a Response Complements Response Inhibition during Performance on a Stop-Signal Task. The Journal of Neuroscience, 29(50), 1587015877. doi:10.1523/JNEUROSCI.3645-09.2009

Cieslik, E. C., Mueller, V. I., Eickhoff, C. R., Langner, R., \& Eickhoff, S. B. (2015). Three key regions for supervisory attentional control: evidence from neuroimaging metaanalyses. Neuroscience \& biobehavioral reviews, 48, 22-34.

Corbetta, M., \& Shulman, G. L. (2002). Control of Goal-Directed and Stimulus-Driven Attention in the Brain. Nature Reviews, 3. 201-215. doi: 10.1038/nrn755

Eickhoff, S., Stephan, K. E., Mohlberg, H., Grefkes, C., Fink, G. R., Amunts, K., \& Zilles, K. (2005). A new SPM toolbox for combining probabilistic cytoarchitectonic maps and functional imaging data. NeuroImage 25(4), 1325-1335

Erika-Florence, M., Leech, R., \& Hampshire, A. (2014). A functional network perspective on response inhibition and attentional control. Nature Communications, 5:4073, doi: $10.1038 /$ ncomms 5073 
Forstmann, B. U., Anwander, A., Schäfer, A., Neumann, J., Brown, S., Wagenmakers, E., ... Turner, R. (2010). Cortico-striatal connections predict control over speed and accuracy in perceptual decision making. Proceedings of the National Academy of Sciences, 107(36), 15916-15920. doi: 10.1073/pnas.1004932107

Friston, K. J., Buechel, C., Fink, G. R., Morris, J., Rolls, E., \& Dolan, R. J. (1997). Psychophysiological and modulatory interactions in neuroimaging. Neuroimage, 6(3), 218-229.

Gur, R. C., Turetsky, B. I., Loughead, J., Waxman, J., Snyder, W., Ragland, J. D., ..., \& Gur, R. E. (2007). Hemodynamic Responses in Neural Circuitries for Detection of Visual Target and Novelty: An Event-Related fMRI Study. Human Brain Mapping, 28. 263274. doi: 10.1002/hbm.20319

Hartwigsen, G., Neef, N. E., Camilleri, J. A., Margulies, D. S., \& Eickhoff, S. B. (2018). Functional Segregation of the Right Inferior Frontal Cyrus: Evidence from Coactivation-Based Parcellation. Cerebral Cortex, bhy049, 1-15. doi:10.1093/cercor/bhy049

Hampshire, A., Chamberlain, S. R., Monti, M. M., Duncan, J., \& Owen, A. M. (2010). The role of the right inferior frontal gyrus: inhibition and attentional control. NeuroImage, 50, 1313-1319. doi:10.1016/j.neuroimage.2009.12.109

Hampshire, A., \& Sharp, D. J. (2015). Contrasting network and modular perspectives on inhibitory control. Trends in Cognitive Sciences, 19(8), 445-452. doi:10.1016/j.tics.2015.06.006

Jaffard, M., Longcamp, M., Velay, J., Anton, J., Roth, M., Nazarian, B., \& Boulinguez, P. (2008). Proactive inhibitory control of movement assessed by event-related fMRI. NeuroImage, 42, 1196-1206. doi:10.1016/j.neuroimage.2008.05.041

Jahfari, S., Stinear, C. M., Claffey, M., Verbruggen, F., \& Aron, A. R. (2010). Responding with Restraint: What are the Neurocognitive Mechanisms? Journal of Cognitive Neuroscience, 22(7), 1479-1492. doi: 10.1162/jocn.2009.21307

Jahfari, S., Verbruggen, F., Frank, M. J., Waldorp, L. J., Colzato, L., Ridderinkhof, K. R., \& Forstmann, B. U. (2012). How Preparation Changes the Need for Top-Down Control of the Basal Ganglia When Inhibiting Premature Actions. The Journal of Neuroscience, 32(32), 10870-10878. doi:10.1523/JNEUROSCI.0902-12.2012 
Langford, Z. D., Krebs, R. M., Talsma, D., Woldorff, M. G., \& Boehler, C. N. (2016). Strategic down-regulation of attentional resources as a mechanism of proactive response inhibition. The European Journal of Neuroscience, 44(4), 2095-103. doi: 10.1111/ejn.13303

Lavallee, C. F., Herrmann, C. S., Weerda, R., \& Huster, R. J. (2014). Stimulus-Response Mappings Shape Inhibition Processes: A Combined EEG-fMRI Study of Contextual Stopping. PLoS ONE, 9(4): e96159. doi:10.1371/journal.pone.0096159

Leunissen, I., Coxon, J. P., \& Swinnnen, S. P. (2016). A Proactive Task Set Influences How Response Inhibition is implemented in the Basal Ganglia. Human Brain Mapping, 37(12), 4706-4717. doi: 10.1002/hbm.23338

Levy, B. J., \& Wagner, A. D. (2011). Cognitive control and right ventrolateral prefrontal cortex: reflexive reorienting, motor inhibition, and action updating. Annals of the New York Academy of Science, 1224, 40-62. doi: 10.1111/j.1749-6632.2011.05958.x

Mirabella, G. (2014). Should I stay or should I go? Conceptual underpinnings of goal-directed actions. Frontiers in Systems Neuroscience, 8. 1-21. doi: 10.3389/fnsys.2014.00206

Miyake, A., Friedman, N. P., Emerson, M. J., Witzki, A. H., \& Howerter, A. (2000). The Unity and Diversity of Executive Functions and Their Contributions to Complex “Frontal Lobe” Tasks: A Latent Variable Analysis. Cognitive Psychology, 41, 49100. doi:10.1006/cogp.1999.0734

O’Reilly, J. X., Woolrich, M. W., Behrens, T. E., Smith, S. M., \& Johansen-Berg, H. (2012). Tools of the trade: psychophysiological interactions and functional connectivity. Social cognitive and affective neuroscience, 7(5), 604-609.

Schmider, E., Ziegler, M., Danay, E., Beyer, L., \& Bühner, M. (2010). Is It Really Robust? Reinvestigating the Robustness of ANOVA Against Violations of the Normal Distribution Assumption. Methodology, 6(4). 147-151. doi: 10.1027/1614$2241 / \mathrm{a} 000016$

Sebastian, A., Jung, P., Neuhoff, J., Wibral, M., Fox, P. T., Lieb, K., ..., Mobascher, A. (2016). Dissociable attentional and inhibitory networks of dorsal and ventral areas of the right inferior frontal cortex: a combined task-specific and coordinate-based metaanalytic fMRI study. Brain Structure and Function, 221(3), 1635-1651. Doi:

10.1007/s00429-015-0994-y 
Shulman, G. L., Astafiev, S. V., Franke, D., Pope, D. L. W., Snyder, A. Z, McAvoy, M. P., \& Corbetta, M. (2009). Interaction of stimulus-driven reorienting and expectation in ventral and dorsal fronto-parietal and basal ganglia-cortical networks. The Journal of Neuroscience, 29(14), 4392-4407. doi:10.1523/JNEUROSCI.5609-08.2009

Singh-Curry, V., \& Husain, M. (2009). The functional role of the inferior parietal lobe in the dorsal and ventral stream dichotomy. Neuropsychologia, 47. 1434-1448. doi:10.1016/j.neuropsychologia.2008.11.033

Swann, N. C., Cai, W., Conner, C. R., Pieters, T. A., Claffey, M. P., George, J. S., ... Tandon, N. (2012). Roles for the pre-supplementary motor area and the right inferior frontal gyrus in stopping action: Electrophysiological responses and functional and structural connectivity. NeuroImage, 59, 2860-2870. doi:10.1016/j.neuroimage.2011.09.049

Swann, N. C., Tandon, N., Pieters, T. A., \& Aron, A. R. (2013). Intracranial electroencephalography Reveals Different Temporal Profiles for Dorsal- and Ventrolateral Prefrontal Cortex in Preparing to Stop Action. Cerebral Cortex, 23. 2479-2488. doi:10.1093/cercor/bhs245

Swick, D., \& Chatham, C. H. (2014). Ten years of inhibition revisited. Frontiers in Human Neuroscience, 8. doi:10.3389/fnhum.2014.00329

van Belle, J., Vink, M., Durston, S., \& Zandbelt, B. B. (2014). Common and unique neural networks for proactive and reactive response inhibition revealed by independent component analysis of functional MRI data. NeuroImage, 103, 65-74. doi:10.1016/j.neuroimage.2014.09.014

Verbruggen, F., Aron, A. R., Band, G. P., Beste, C., Bissett, P. G., Brockett, A. T., ... \& Colzato, L. S. (2019). A consensus guide to capturing the ability to inhibit actions and impulsive behaviors in the stop-signal task. Elife, 8, e46323.

Verbruggen, F., Aron, A. R., Stevens, M. A., \& Chambers, C. D. (2010). Theta burst stimulation dissociates attention and action updating in human inferior frontal cortex. Proceedings of the National Academy of Sciences, 107(31), 13966-13971. doi: 10.1073/pnas.1001957107

Verbruggen, F., Chambers, C. D., \& Logan, G. D. (2013). Fictitious Inhibitory Differences: How Skewness and Slowing Distort the Estimation of Stopping Latencies. Psychological Science, 24(3), 352-362. doi: 10.1177/0956797612457390 
Verbruggen, F., \& Logan, G. D. (2008). Response inhibition in the stop-signal paradigm. Trends in Cognitive Sciences, 12(11), 418-424. doi:10.1016/j.tics.2008.07.005

Verbruggen, F. \& Logan, G. D. (2009a). Models of Response Inhibition in the Stop-Signal and Stop-Change Paradigms. Neuroscience \& Biobehavioural Reviews, 33(5), 647661. doi:10.1016/j.neubiorev.2008.08.014

Verbruggen, F. \& Logan, G. D. (2009b). Proactive Adjustments of Response Strategies in the Stop-Signal Paradigm. Journal of Experimental Psychology: Human Perception and Performance, 35(3), 835-854. doi: 10.1037/a0012726

Vogt, B. A. (2016). Midcingulate cortex: structure, connections, homologies, functions and diseases. Journal of chemical neuroanatomy, 74, 28-46.

Wessel, J. R., Conner, C. R., Aron, A. R., \& Tandon, N. (2013). Chronometric electrical stimulation of right inferior frontal cortex increases motor braking. Journal of Neuroscience, 33(50), 19611-19619. doi: 10.1523/JNEUROSCI.3468-13.2013

Zandbelt, B. B., Bloemendaal, M., Neggers, S. F. W., Kahn, R. S., \& Vink, M. (2013). Expectations and Violations: Delineating the Neural Network of Proactive Inhibitory Control. Human Brain Mapping, 34, 2015-2024. doi:10.1002/hbm.22047

Zandbelt, B. B., \& Vink, M. (2010). On the Role of the Striatum in Response Inhibition. PLoS ONE, 5(11). e13848. doi:10.1371/journal.pone.0013848 